\title{
非负方阵不可约性和非周期性判定与算法 ${ }^{*}$
}

\author{
周 作 领 \\ (中山大学岭南(大学)学院,广州 510275) \\ 肖 金庐 \\ (中山大学计算中心,广州 510275)
}

\section{关镜词非负方阵、不可约性、非周期性}

不可约性和非周期性 (本原性) 是非负方阵理论两个基本概念. 据作者所知, 如何判定给 定非负方阵的不可约性和非周期性尚无可行算法, 特别对后者. 通常,给定一个 $k \times k$ 阶非负 方阵, 可以构造一个相联系的有向图, 它的顶点由 $k$ 个元素组成, 而有向弧则由方阵的非元 素决定 ${ }^{\omega}$. 本文将对给定非负方阵引进一个新的有向图, 并由此分别给出不可约性和非周期 性的判定(等价)条件和可行算法.

设 $A-\left(a_{i j}\right)$ 为 $k \times k$ 阶非负方阵, 即 $a_{i j} \geqslant 0,1 \leqslant i, j \leqslant k, k \geqslant 1$. 对 $n>0$, 记 $A^{n}-\left(a_{i j}^{(i)}\right)$, 它也是非负方阵. $A$ 称作不可约的, 如果对固定的 $1 \leqslant i, j \leqslant k$, 存在 $n>0$, 使 $a_{i j}^{(i)}>0 . A$ 称作非周期的 (本原的), 如果存在 $n>0$, 使 $A^{*}>0$, 即 $a_{i j}^{(n)}>0, \forall 1 \leqslant i$, $j \leqslant k$. 非周期性蕴含不可约性, 但反之不然. 引进与 $A$ 相联系的有向图 $G(A)$ 如下. $G(A)$ 的顶点由 $A$ 的非零元索组成; 从 $G(A)$ 的顶点 $a_{i j}$ 到顶点 $a_{l m}$ 有一条有向弧, 如果 $j-l$. 设 $a_{i_{i_{0}} i_{1}} \rightarrow a_{i_{1} i_{3}} \rightarrow \cdots \rightarrow a_{i_{n} i_{n+1}}$ 是 $G(A)$ 的一条有向路径, 则出现在其中的有向弧个数叫作它的 步长. 有向闭路的步长叫作它的周期.

本文下面恒设 $A-\left(a_{i j}\right)$ 为 $k \times k$ 阶非负方阵 $(k \geqslant 1)$, 并假设 $A$ 的每一行和每一列都 至少有一个元素不为零. 我们的主要结果是

定理 $1 A$ 不可约 $\Leftrightarrow G(A)$ 有一条闭路过所有顶点.

它理 $2 A$ 非周期 $\Leftrightarrow A$ 不可约且 $G(A)$ 有两条闭路周期互素.

先证明下述三个引理:

引理 1 设 $n \geqslant 1,1 \leqslant i, j \leqslant k$, 则 $a_{i j}^{(n)}>0 \Leftrightarrow$ 存在 $1 \leqslant i-i_{1}, i_{2}, \cdots, i_{n} \leqslant k$, 使 $a_{i i_{1}} \rightarrow a_{i_{1} i_{s}} \rightarrow \cdots \rightarrow a_{i_{e} ;}$ 是 $G(A)$ 的有向路径,步长为 $n-1$.

对方阵乘法归纳证明, 从略.

引理 1 的推论 设 $n \geqslant 1,1 \leqslant i \leqslant k$, 则 $a_{i i}^{(i)}>0 \Leftrightarrow$ 存在顶点 $a_{i j}>0(1 \leqslant i \leqslant k)$ 和从 $a_{i j}$ 到 $a_{i j}$ 周期为 $n$ 的有向闭路.

证 据假设, 存在 $1 \leqslant i \leqslant k$, 使 $a_{i j}>0$. 在引理 1 中, 取 $j-i$, 并注意到 $a_{i n i} \rightarrow a_{i i}$ 是有向路径即可.

设 $B-\left(b_{i j}\right)$ 亦是 $k \times k$ 阶非负方阵. 记 $A \gg B$, 如果 $a_{i j}=0 \Rightarrow b_{i j}=0, \forall 1 \leqslant i, j \leqslant k$.

本文1991年4月22 日收到. 1991 年10月18日收到修改稿.

- 国家自然科学基金和国家数娄基金资助项目. 
引理 2 设 $A$ 和 $B$ 是 $k \times k$ 非负方阵. 若 $b_{i i}>0, \forall 1 \leqslant i \leqslant k$, 则 $B A \gg A$. 由方阵乘法直接证明.

引理 3 设 $p$ 和 $q$ 是两个正整数, 且 $d(p, q)-1$, 即 $p$ 和 $q$ 互素. 则对任意正整数 $c$, 存在正整数 $\lambda$ 和 $\mu$, 使 $d(p+\lambda c, q+\mu c)=1$.

证 设 $d(p, q)-1$, 则存在非雾整数 $s$ 和 $t$, 使 $s p+t q-1$. 易见 $s \cdot t<0$. 设 $s<0$, 则有 $s(p+t c)+t(q-s c)-s p+t q=1$. 令 $\lambda=t$ 和 $\mu--s$ 、我们有 $d(p+$ $\lambda c, q+\mu c)-1$.

定理 1 的证明: $\Longrightarrow$ 部分. 显然只需证明对 $G(A)$ 的任意两个顶点 $a_{i l}$ 和 $a_{i m}$, 存在从 $a_{i l}$ 到 $a_{i m}$ 的有向路径即可. 据假设存在 $n \geqslant 1$, 使 $a j_{j}^{n}>0$. 据引理 1 , 存在有向路径 $a_{l_{3}} \rightarrow \cdots \rightarrow a_{l_{n} i}$. 显然 $a_{i l} \rightarrow a_{l_{1}} \rightarrow \cdots \rightarrow a_{i_{n} i} \rightarrow a_{i_{m}}$ 亦是有向路径.

$\Longleftarrow$ 部分. 任给 $1 \leqslant i, i \leqslant k$. 因为 $A$ 的每一行和每一列都至少存在一个非忽元素, 故 存在 $1 \leqslant l, m \leqslant k$, 使得 $a_{i l}>0$ 和 $a_{m i}>0$. 据假设存在从 $a_{i l}$ 到 $a_{m i}$ 的有向路径, 并设其 步长为 $n \geqslant 1$. 据引理 1 , 我们有 $a_{i j}^{(p+1)}>0$. 定理 1 证毕.

定理 2 的证明: $\Longrightarrow$ 部分. 设 $n \geqslant 1$, 使 $A^{n}>0$. 显然 $A^{n+1}>0$. 由 $a_{11}^{(n)}>0$ 和 $a_{11}^{(n+1)}>$ 0 , 据引理 1 , 存在有向路径 $a_{1 i_{2}} \rightarrow \cdots \rightarrow a_{i_{n 1}}$ 和 $a_{1 l_{2}} \rightarrow \cdots \rightarrow a_{i_{n+1}}$. 据 $A$ 的不可约性, 存在 有向路径 $a_{i_{n 1}} \rightarrow P \rightarrow a_{1 i_{2}}$, 其中 $P$ 是有向路径. 显然, $a_{l_{n+1}} \rightarrow P \rightarrow a_{l_{2}}$ 也是有向路径. 易见 $a_{1 i_{2}} \rightarrow \cdots \rightarrow a_{i_{n 1}} \rightarrow P \rightarrow a_{1 i_{2}}$ 和 $a_{1 l_{2}} \rightarrow \cdots \rightarrow a_{l_{n+1^{1}}} \rightarrow P \rightarrow a_{1 l_{2}}$ 是两条有向闭路且周期互素.

$\Longleftarrow$ 部分. 设 $P$ 和 $Q$ 是 $G(A)$ 的两条有向闭路, 周期分别为 $p>0$ 和 $q>0$ 且 $d(p$, $q)-1$. 令 $T$ 是 $G(A)$ 的任一条过所有顶点的闭路, 周期为 $t>0$. 据引理 3, 存在正整数 2 和 $\mu$, 使 $d(p+\lambda t, q+\mu t)=1 . P$ 和 $T$ 有公共顶点, 故由 $T$ 重复 $\lambda$ 次和 $P$ 可得过所有顶点 的另一条有向闭路, 周期为 $p+\lambda t$. 同理存在另一条过所有顶点的有向闭路, 周期为 $q+\mu t$. 据互素整数的基本性质, 不妨设存在整数 $s>0$ 和 $r>0$, 使 $s(p+\lambda t)=r(q+\mu t)+1$. 又据 $A$ 的不可约性, 存在整数 $h>0$, 使 $A+A^{2}+\cdots+A^{k}>0$. 下面我们证明 $A^{a(p+\alpha h)}>0$.

设 $g_{1}$ 和 $g_{2}$ 为任意正整数. 显然对任意顶点 $a_{i j}>0$, 存在过 $a_{i j}$ 的一条有向闭路, 周 期为 $g_{1} s(p+\lambda t)$. 据引理 1 的推论, 我们有 $a_{1}^{\left.\left(g_{1}(x)+\lambda t\right)\right)}>0$. 因为 $A$ 的每一行和每一列至少 存在一个非零元素, 故 $a_{i j}^{\left(p_{1} s(p+2 t)\right)}>0, \forall 1 \leqslant i \leqslant k$, 即 $A^{x_{1}(p+2 t)}$ 的主对角线上元素均大于 零. 对 $A^{\left.z_{1}(q)+\mu t\right)}$ 和 $A^{z_{1}(p+\lambda t)} \cdot A^{z_{2}(q+\mu t)}$ 有同样的结论. 据引理 2, 我们有

$$
\begin{gathered}
A^{h(p+2 t)}=A^{(k-1))(p+\lambda t)} \cdot A^{r(q+\mu t)} \cdot A \gg A, \\
A^{h(p+\lambda t)}=A^{(k-2)(p+\lambda t)} \cdot A^{2 r(q+\mu t)} \cdot A^{2} \gg A^{2}, \\
\vdots \\
A^{h(p+\lambda t)}=A^{h t(q+\mu t)} \cdot A^{k} \gg A^{k} .
\end{gathered}
$$

由 $A+A^{2}+\cdots+A^{k}>0$ 即得 $A^{k(p+2 t)}>0$. 定理 2 证毕.

据定理 1 和定理 2 , 判定给定非负方阵的不可约性和非周期性归结为判定相联系的 有向 图的过所有顶点的有向闭路的存在性和有向闭路的互素周期的存在性. 据此, 我们可以给出 可行算法.

算法：从略.

\section{考文嗝}

[1] Berman, A. and Plemmons, R. J., Nonnegative Matrices in the Mathematical Sciences, Academic Press, New York, San Francisco, London, 1979. 